\title{
Archéopages
}

Archéopages

Archéologie et société

\section{Campement paléolithique supérieur en bord de} Seine

\section{Miguel Biard et Cyril Marcigny}

\section{(2) OpenEdition}

1 Journals

Édition électronique

URL : https://journals.openedition.org/archeopages/322

DOI : 10.4000/archeopages.322

ISSN : 2269-9872

\section{Éditeur}

INRAP - Institut national de recherches archéologiques préventives

\section{Édition imprimée}

Date de publication : 1 mai 2013

Pagination : 96-97

ISSN : 1622-8545

\section{Référence électronique}

Miguel Biard et Cyril Marcigny, « Campement paléolithique supérieur en bord de Seine », Archéopages

[En ligne], 35 | 10/2012, mis en ligne le 01 octobre 2014, consulté le 22 janvier 2022. URL : http:// journals.openedition.org/archeopages/322 ; DOI : https://doi.org/10.4000/archeopages.322 



\title{
Campement paléolithique supérieur en bord de Seine
}

\author{
Miguel Biard \\ Inrap, étude des sites paléolithiques \\ Cyril Marcigny \\ Inrap, UMR 6566 «Centre de Recherche en Archéologie, \\ Archéosciences, histoire», responsable d'opération
}

Site

Alizay et Igoville

Eure

Date

Février 2011-août 2012

Superficie

14 ha

Équipe

$\mathscr{8}$

Responsables de secteur Bruno Aubry

Étude faunique

Céline Bémili
Le site d'Alizay est implanté en zone de convergence du lit majeur actuel et de la basse terrasse de la Seine. Il se trouve sur la rive droite, au niveau de l'actuelle confluence de ce fleuve et de l'Eure. Durant la Pré- et la Protohistoire, cette zone humide était parcourue de chenaux autour desquels se sont installés les hommes. La fouille fait suite à un diagnostic archéologique de 37 ha réalisé en 2009 (Aubry, 2010), motivé par l'agrandissement d'une carrière de granulat (Lafarge Seine-Nord et Cemex). Elle a pour objectif de comprendre les modes d'occupation du sol par les divers groupes humains ayant fréquenté cette zone prescrite sur un premier secteur, tandis qu'une deuxième zone de fouille de 2 ha devrait suivre, puis une dernière de 14 ha.

La position du site dans un secteur régulièrement inondé a favorisé la fossilisation et la conservation des témoins archéologiques sous d'épaisses couches de terre formant une importante stratigraphie de 3 à $4 \mathrm{~m}$ de profondeur. Les sols des différentes époques ont ainsi été préservés d'une trop forte érosion et la plupart des vestiges et des structures sont très bien conservés. La compréhension générale du site est donc autant planimétrique que stratigraphique. Chaque occupation est ainsi replacée dans son contexte environnemental, hydrologique et topographique. La reconstitution de la géométrie des sols sur le temps long forme l'un des axes de recherche privilégiée pour assurer une bonne compréhension des différentes occupations humaines et surtout des relations homme-milieux (évolutions, interactions, etc.). Dans un tel cadre, les approches multiscalaires conduites par une équipe interdisciplinaire constituent la clef de voûte de cette étude. Ces travaux prennent appui sur les outils actuellement à notre disposition : intégration des données via un système d'information géographique (SIG), imagerie $3 \mathrm{D}$ (jusqu'à la constitution de blocs diagrammes), études paléoenvironnementales couplées à l'analyse intégrée des données archéologiques, etc.

Parmiles nombreuses occupations identifiées, la fouille a permis de mettre au jour, sur les niveaux attribués à l'extrême fin du Paléolithique supérieur (au-dessus de niveaux du Dryas récent), une zone dense en vestiges [ill. 1] d'une superficie d'environ $70 \mathrm{~m}^{2}$ qui a livré plus de 4500 pièces lithiques [ill. 3] et osseuses [ill. 2] (le locus 28 704). Au sein du corpus lithique, il faut noter d'une part la présence conjointe d'un débitage laminaire et lamellaire de qualité et d'une production de petites lames larges, supports d'armatures, d'autre part la découverte d'armatures inédites en Normandie. Outre la qualité de l'assemblage
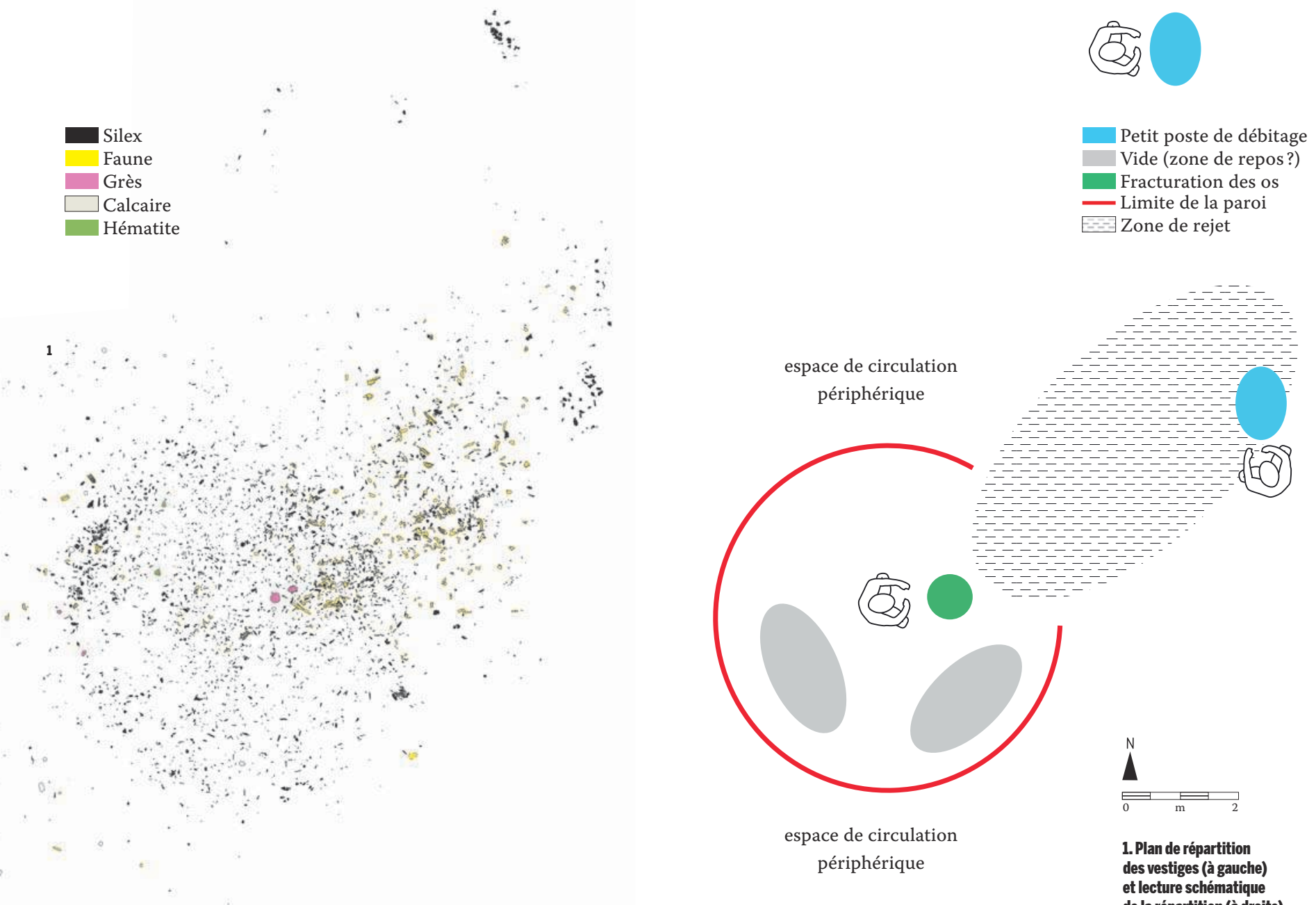

de la répartition (à droite). 
lithique, la spécificité du site réside également dans la présence de restes fauniques bien conservés, un calage stratigraphique cohérent ainsi qu'un excellent état de conservation.

Le locus 28704 appartient à une période chronologique charnière pour laquelle les témoignages sont à ce jour très ténus en Haute-Normandie: la transition entre le Dryas récent et le début du Préboréal. Cette occupation tardiglaciaire de la vallée de la Seine présente une production lithique singulière dont les grandes tendances se retrouvent dans les faciès culturels contemporains (épiAhrensbourgien, Belloisien, Laborien), mais auxquels viennent s'ajouter des éléments de diagnoses très intéressants, voire inédits en France. L'état de conservation remarquable dulocus, lié au contexte stratigraphique de berge, permet une lecture fine de la répartition spatiale des vestiges. Elle forme une concentration constituant un cercle presque parfait de $7 \mathrm{~m}$ de diamètre auquel se greffe, au nord-est, une zone de rejet de $8 \mathrm{~m}^{2}$ qui renferme une grande majorité des restes fauniques. Au sein du cercle, légèrement décentré vers l'est, les occupants ont abandonné une enclume ainsi que le percuteur qui semble avoir servi à fracturer des os comme le montrent les nombreux stigmates de fracturation intentionnelle. Seul le genre Bos est à ce jour déterminé dans le spectre faunique. Mais la découverte d'un fragment de bois de renne vient alimenter la question de la présence de cet animal au nord de la France après 12300 avant notre ère (Fontana, 2012).

Toujours au centre du cercle, les amas de tailles ont été déplacés, poussés sur les bords, créant d'incontestables effets de paroi dont les limites viennent étayer l'hypothèse de la présence d'une structure d'habitat. Tente, hutte ou auvent, l'étude spatiale des vestiges (en cours) permettra peut-être d'apporter des réponses. Au nord-est du locus, sont identifiés deux postes de débitage renfermant les déchets de diminution d'un nucléus laminaire (successions de tablettes et de produits d'entretien). Cet objectif de taille est un caractère chronoculturel important et se traduit par la présence conjointe d'une production de grandes lames régulières et de petites armatures issues d'une production de lamelles. Les premières, de grandes dimensions, semblent avoir été utilisées brutes ou parfois transformées en outils. Les secondes sont réservées à la fabrication d'armatures de traits. Leur obtention au percuteur de pierre tendre demande une bonne gestion du volume et des procédés techniques élaborés, ce dont témoignent

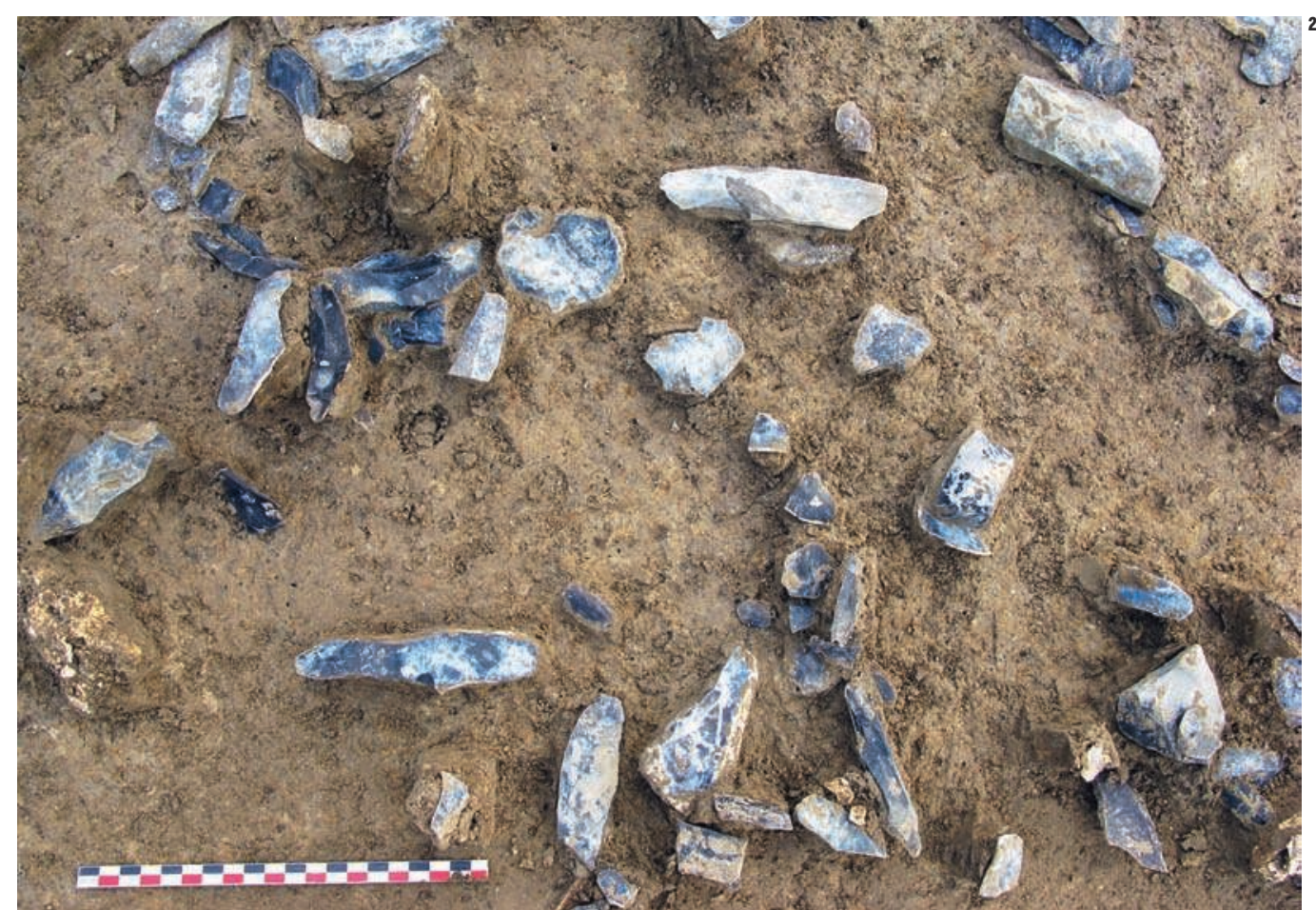

les nombreux remontages. Ce mode de débitage révèle un savoir-faire technique élevé, avec des objectifs précis pour la production de supports laminaires standardisés. Ces caractères rappellent ceux du site du Buhot à Calleville, situé dans la même région (Biard, Hinguant, 2011). L'outillage est représenté en priorité par les grattoirs dont l'étude tracéologique (en cours) viendra sans doute confirmer l'utilisation pour le travail de la peau. Les pièces esquillées sont elles aussi bien représentées et une étude expérimentale est également prévue afin de reproduire les stigmates et comprendre l'utilisation de ces pièces. Quant aux armatures, elles sont de types inédits à ce jour en Normandie, associant des bi-troncatures et des pointes des Blanchère. Les premières sont réalisées sur des lames fines et régulières aux bords parallèles et à profil rectiligne et présentant deux troncatures obliques ou droites. Les secondes réclament comme support des lamelles étroites et rectilignes et présentent un dos droit effectué par retouche croisée. Cette association d'armatures a été récemment observée sur des sites de l'ouest de la France (Naudinot, Jacquier, 2009) et la découverte d'Alizay témoigne de son extension.

Insistant sur l'homogénéité de l'assemblage lithique du site et sur son état de conservation, ce premier survol de l'industrie lithique évoque une production en tout point singulière Outre les caractères décrits, la volonté des tailleurs de produire des lamelles étroites les conduit parfois ainsi
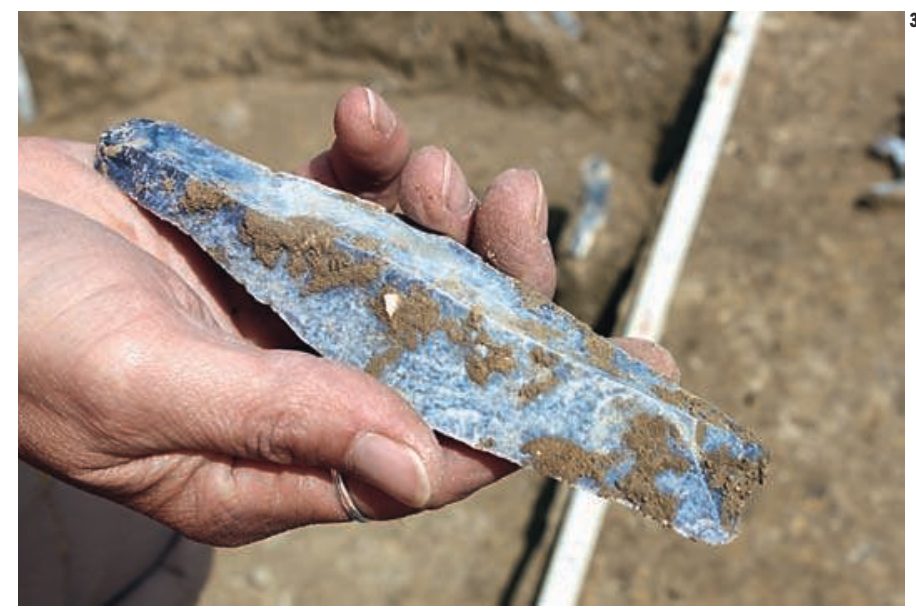

2. Détail des restes osseux

mêlés aux produits

de débitage.

3. Grande lame de première

intention.

à l'emploi opportuniste de nervures à partir d'éclats ou de portions de grandes lames pour produire ces supports et non pas à la seule réduction de nucléus aux volumes souvent limités. Ce sont ces lamelles qui sont ensuite utilisées pour la fabrication de microlithes. Ces caractères originaux alimentent notre connaissance sur les groupes de la fin du Paléolithique et de la naissance du Mésolithique, la position stratigraphique du locus plaçant l'occupation à l'interface entre le Dryas récent et le Préboréal. Une première datation $\mathrm{au}^{14} \mathrm{C}$ vient d'ailleurs confirmer le caractère $317897: 9890+/-40$ avant notre ère). transitoire de cette occupation (Beta

\section{Références bibliographiques}

Aubry B., Aoustin D. (COllab.), Bemilli C. (Collab.), Biard M. (COllab.), Thomann A. (COllab.), 2010, Igoville (Eure), de -10 ooo à moins 3 mètres, Rapport de diagnostic, Inrap Grand-Ouest, SRA HauteNormandie, $165 \mathrm{p}$.

Biard M., Hinguant S., Beurion C. (Collab.), Deloze V. (Collab.), Forre P. (Collab.), Sellami F. (COLlAB.), 2011, Le bivouac préhistorique du Buhot à Calleville (Eure), caractérisation d'un assemblage lithique lamino-lamellaire de la fin du Paléolithique supérieur,

Recherches archéologiques, 2, Inrap/ CNRS éditions, $168 \mathrm{p}$

NAUDINOT N., JACQUIER J., 2009, «Un site tardiglaciaire en place à la Fosse (Villiers-Charlemagne, Mayenne) : premiers résultats et implications chronoculturelles », Bulletin de la Société préhistorique française, 106, p. $145-158$. 